\title{
Effects of Specific Type of Exercises on QOL in the Elder with MCI
}

\author{
Seung youn Hong* \\ Department of Senior Industry, Kangnam University, South Korea
}

Received: January 24, 2018; Published: February 02, 2018

*Corresponding author: Seung youn Hong, Dept of Senior Industry, Kangnam University, Yongin, South Korea, Tel: +81-10-8799-4185; Email: Yoni91@hotmail.com

\begin{abstract}
Background: The purpose of this research was to identify the relationship between major exercises (aerobic, resistance, and walking) participation and the perception of problems in each dimension of the health-related quality of life assessed by the EuroQOL (EQ-5D) in community-dwelling older adults with mild cognitive impairment (MCI)
\end{abstract}

Method: A cross-sectional study was conducted with 755 elders (mean age: $76.03+6.26$, range: $65-93$ ) with MCI (Mean MMSE 23.68+4.16) who were recruited from local senior centers. Demographic factors, exercise questionnaire, five EuroQOL subsets (mobility, self-care, usual activities, pain/discomfort, and anxiety/depression) were investigated. The independent association between each exercise and five QOL subsets were determined using odds ratios (OR) adjusted for three demographics (age, sex), using multivariate logistic analysis.

Results: Subjects with problems in mobility dimension performed less exercise of resistance exercise and walking than those with normal mobility (resistance: OR, 0.633; 95\% CI: 0.457-0.877; walking: OR, 0.538; 95\% CI: 0.396-0.732). Subjects with problems in self-care performed less walking than those with normal self-care (Or, 0.551; $95 \%$ CI: $0.367-0.827)$. Subjects with difficulties in a usual activity performed less walking than those with normal self-care (OR, 0.715; 95\% CI: 0.521-0.980). Subjects with problems in anxiety/depression performed less walking than those with normal anxiety/depression (OR, 0.641; 95\% CI: 0.473-0.868)

Conclusion: Among the QOL dimension, mobility, self-care, usual activity, and anxiety/depression were independently associated with walking exercise in this older with MCI. This suggests that engaging in regular walking is important for achieving higher QOL in the elders with MCI.

Keywords: MCI; Exercise; QOL

Abbreviations: MCI: Mild cognitive impairment; OR: Odds Ratios; QOL: Quality of Life; IADL: Instrumental Activities of Daily Living; IPAQ: International Physical Activity Questionnaire; BMI: Body Mass Index

\section{Introduction}

The rapid aging of the population involves an increase in the prevalence among the elders of mild cognitive impairment Hood [1]. Mild cognitive impairment (MCI) is an intermediate state between the cognitive changes of normal aging and dementia. Individuals with mild cognitive impairment (MCI) are at high-risk group for developing dementia-a rate of 10 to $15 \%$ per year compared with $1 \%$ to $2 \%$ per year among the general population Petersen, Doody, et al. [2] and the prevalence of global MCI is estimated ranges from $9.6 \%$ to $21.6 \%$ Jia et al. [3]; Lara et al. [4], Limongi et al. [5]. Given that this cohort is at high risk of developing dementia and lowering quality of life, it is important to identify effective strategies to manage the cognitive decline and maintain the quality of life. Physical activity and exercise as the nonpharmacological treatment for MCI and dementia have received considerable attention. A meta-analysis of 29 randomized control studies, the seniors with MCI demonstrate improvement in cognitive function following an exercise program that combines strength training with aerobic exercise Smith, Blumenthal, et al. [6].

Another study has also indicated that the long-term effect of exercise in delaying the onset of dementia for years PaillardBorg et al. [7], Nascimento et al. [8]. However, only a few studies analyzed the effects among MCI patients of the extended outcomes of physical exercise on quality of life Chen et al. [9]; Lautenschlager et al. [10], Chen et al. [9] reported a 12-week exercise training including moderate-intensity aerobic exercise, strength exercise, and balance exercise was correlated with QOL. Further, only a few studies analyzed the effects of specific types of exercise on Quality of life (QOL) among the elders with MCI. Therefore, the aim of 
the present study was to identify the relationship between three types of exercise participation more related to the perception of problems in each dimension of the health-related quality of life (HRQoL) assessed by the EQ-5D dimensions 3 level questionnaire in community-dwelling older adults with MCI.

\section{Materials and Methods}

\section{Subjects}

A cross-sectional study was conducted with 755 elders with MCI (590 females and 185 males aged 62-93 years). The subjects were recruited from 38 local senior centers in Seoul, South Korea. Subject's eligibility criteria were:

a) 65 years or older,

b) A score of 20-24 on the MMSE-K (Mini-Mental State Examination-Korea)Park \& Kwon [11],

c) Able to communicate,

d) Absence of dementia, according to DSM-IV,

e) Maintain activities of daily living or slight impairment in instrumental activities of daily living (IADL), as defined by no more than one item of IADL changes and

f) Completion of a written consent form.

\section{Procedures}

Participants were assessed by the EQ-5D questionnaire and physical activity questionnaire. The EuroQOL five-dimension questionnaire (EQ-5D) consists of five dimensions including selfcare, mobility, usual activities, pain/discomfort, and anxiety/ depression, each of which offered three possible responses: no problems/ some or moderate problems/ extreme problems(EQ$5 D, 2016)$ [12]. The responses to each EQ-5D dimensions were collapsed into a two-tier variable: In each EQ-5D dimension, "no problem" was considered as normal, whereas "some problems" or "severe problems" were considered problematic. The level of PA was measured by asking participants how often they engaged in exercise each week including a Korean version of the international physical activity questionnaire (IPAQ). Subjects who performed moderate intensity of aerobic exercise were defined 150 minutes of aerobics per week. Subjects who performed resistance exercise was Table 1: General characteristics of participants by gender $(n=775)$. defined those who performed exercises such as push-ups, crunches, or chin-ups for 30 minutes per session at least two times in the past week. Walking was defined as persons who walked more than one day in the past week at least 10 minutes in each session. Body mass index (BMI) was calculated as weight in kg divided by the square of height in meter.

\section{Statistical Analysis}

Descriptive statistics were used to evaluate the distribution of age, sex, BMI, MMSE and EQ-5D dimension. Chi-square test was conducted to detect the association between three types of exercise participation and age, sex, BMI, MMSE and problems in the five EQ-5D dimensions. Independent association between each type of exercise and the five EQ-5D dimensions were determined using odds ratio (OR) adjusted for two demographic factors (age, MMSE) on multivariate logistic regression analysis. IBM SPSS statistics version 20.0 (IBM Corporation, Armonk, NY, USA) was used for all the analyses. Values $\mathrm{p}<0.05$ were considered statistically significant.

\section{Results \\ Demographics}

The general characteristics of participants were shown in Table 1. The study population included 185 men (23.8\%) and 562 women (76.2\%) age 65 and older. The average age of study population was 76.03 years (SD: 6.26, ranging: 65-93). The mean age of men and women was 77.74 and 76.28 years, respectively. There was a significant difference in mean age between men and women $(t=2.846, p<0.001)$. The majority of the subjects have low education levels (54.3\%), and women were more likely than men to have less education $(\mathrm{p}<.001)$. The majority of the subject has rated their health as over fair (66.3\%), and there was no significant difference In self-rated health between men and women. The average MMSE-K was 23.68 , and there was no gender difference. The proportions of older participants who performed the moderate aerobic exercise, resistance, or walking exercises were $24.8 \%, 30.5 \%$, or $63 \%$, respectively. Men were more likely to perform resistance exercise than women ( $47.2 \%$ vs. $26.0 \%, \mathrm{p}<.001)$. Each EQ-5D dimension differently affected by gender. Women were more likely to have problem in two type of EQ-5D dimension (mobility: $49.7 \%$ vs. $41.7 \%, p=0.043$; pain/discomfort: $70.9 \%$ vs. $58.9 \%, p=0.003$ ) than men (Table 1).

\begin{tabular}{|c|c|c|c|c|c|}
\hline Variable & Classification & Total Mean or n(\%) & M Mean (SD) or n(\%) & W Mean (SD) or n(\%) & t or X2 (p-value) \\
\hline Gender & & & $185(23.8)$ & $562(76.2)$ & \\
\hline Age(yr) & & $76.03(6.26)$ & $77.74(5.53)$ & $76.28(5.89)$ & $2.846(<.001)$ \\
\hline \multirow{4}{*}{ Education } & <elementary & $421(54.3)$ & $50(11.9)$ & $370(88.1)$ & \multirow{4}{*}{$75.21(<.001)$} \\
\hline & Middle & $160(20.6)$ & $33(20.6)$ & $127(79.4)$ & \\
\hline & High & $135(17.4)$ & $49(36.3)$ & $86(63.7)$ & \\
\hline & $>$ college & $59(7.6)$ & $31(52.5)$ & $28(47.5)$ & \\
\hline \multirow{3}{*}{ Self-rated health } & Excellent & $17(2.2)$ & $3(17.6)$ & $14(82.4)$ & \multirow{3}{*}{$8.258(0.083)$} \\
\hline & Good & $132(17.1)$ & $38(28.8)$ & $94(71.2)$ & \\
\hline & Fair & $363(47.0)$ & $74(28.4)$ & $288(79.6)$ & \\
\hline
\end{tabular}




\begin{tabular}{|c|c|c|c|c|c|}
\hline & Poor & $213(27.6)$ & 42(19.7) & $171(80.3)$ & \\
\hline & Very poor & $47(6.1)$ & $5(10.5)$ & $42(29.4)$ & \\
\hline BMI $\left(\mathrm{Kg} / \mathrm{m}^{2}\right)$ & & $23.60(3.05)$ & $23.07(2.72)$ & $23.75(3.12)$ & $-2.502(0.013)$ \\
\hline MMSE-K & & $23.68(4.16)$ & $23.53(3.58)$ & $22.95(3.94)$ & $1.701(0.089)$ \\
\hline \multicolumn{6}{|l|}{ EQ5D } \\
\hline \multirow{2}{*}{ Mobility, n(\%) } & Normal & $402(51.9)$ & $95(58.3)$ & $306(50.3)$ & \multirow{2}{*}{$3.258(0.013)$} \\
\hline & Problem & $372(48.1)$ & $68(41.7)$ & $302(49.7)$ & \\
\hline \multirow{2}{*}{ Self-care, n (\%) } & Normal & $656(84.6)$ & $135(82.8)$ & $520(85.1)$ & \multirow{2}{*}{$0.516(0.272)$} \\
\hline & Problems & $119(15.4)$ & $28(17.2)$ & $91(14.9)$ & \\
\hline \multirow{2}{*}{ Usual Activities, n (\%) } & Normal & $502(64.6)$ & $102(62.6)$ & $399(65.3)$ & \multirow{2}{*}{$0.419(0.285)$} \\
\hline & Problems & $275(35.4)$ & $61(37.4)$ & $212(34.7)$ & \\
\hline \multirow{2}{*}{$\begin{array}{c}\text { Pain/Discomfort, } \mathrm{n} \\
(\%)\end{array}$} & Normal & $245(31.5)$ & $67(41.1)$ & $178(29.1)$ & \multirow{2}{*}{$8.524(0.003)$} \\
\hline & Problems & $532(68.5)$ & $96(58.9)$ & $433(70.9)$ & \\
\hline \multirow{2}{*}{$\begin{array}{c}\text { Anxiety/depression, } \\
\text { n (\%) }\end{array}$} & Normal & $428(55.1)$ & $95(58.3)$ & $331(54.3)$ & \multirow{2}{*}{$0.840(0.204)$} \\
\hline & Problems & $349(44.9)$ & $68(41.7)$ & $279(45.7)$ & \\
\hline \multirow{2}{*}{ Moderate Aerobic } & Non-exs & & $122(74.8)$ & $460(75.3)$ & \multirow{2}{*}{$0.013(0.49)$} \\
\hline & Exs & & $41(25.2)$ & $151(24.7)$ & \\
\hline \multirow{2}{*}{ Resistance } & Non-exs & & $86(52.8)$ & $452(74.0)$ & \multirow{2}{*}{$27.329(<0.001)$} \\
\hline & Exs & & $77(47.2)$ & $159(26.0)$ & \\
\hline \multirow{2}{*}{ Walking } & Non-exs & & $52(31.9)$ & $234(38.3)$ & \multirow{2}{*}{$2.259(0.079)$} \\
\hline & Exs & & $111(68.1)$ & $377(61.7)$ & \\
\hline
\end{tabular}

\section{Independent effects of each variable on exercise}

Table 2: Subjects characteristics by exercise type.

\begin{tabular}{|c|c|c|c|c|c|c|c|c|c|c|c|}
\hline Variable & \multicolumn{2}{|c|}{ Classification } & $\begin{array}{c}\text { NON-AER } \\
\text { EXS }\end{array}$ & AER EXS & $\operatorname{tor} X^{2}$ & NON-EXS & $\begin{array}{l}\text { RES } \\
\text { EXS }\end{array}$ & $\operatorname{tor} \mathrm{X}^{2}$ & $\begin{array}{c}\text { Non- } \\
\text { Walking }\end{array}$ & Walking & $\operatorname{tor} X^{2}$ \\
\hline Age & & & $75.10(5.89)$ & $77.06(5.75)$ & $-2.56^{* *}$ & $76.90(5.88)$ & $75.58(5.65)$ & $2.46^{*}$ & $76.80(5.82)$ & 76.11(5.88) & 1.56 \\
\hline \multirow{2}{*}{ sex } & Male & & $102(62.6)$ & $61(37.4)$ & \multirow{2}{*}{1.87} & $105(66.5)$ & $53(33.5)$ & \multirow{2}{*}{$26.03^{* *}$} & $102(62.6)$ & $61(37.4)$ & 1.87 \\
\hline & Female & & $417(68.2)$ & $194(31.8)$ & & $501(84.5)$ & $92(15.5)$ & & $417(68.2)$ & $194(31.8)$ & \\
\hline BMI & & & $23.56(3.15)$ & $23.68(3.15)$ & -0.51 & $23.72(3.11)$ & $23.13(2.87)$ & $2.06^{*}$ & $23.56(3.15)$ & $23.68(2.85)$ & -0.51 \\
\hline MMSE & & & $22.79(4.08)$ & $23.65(3.34)$ & $-2.93^{*}$ & $22.83(3.92)$ & $23.97(3.45)$ & $-3.22^{* *}$ & $22.79(4.08)$ & $23.65(3.34)$ & $-2.93^{*}$ \\
\hline \multirow{10}{*}{ EQ-5D } & \multirow{2}{*}{ Mobility } & Normal & $240(59.7)$ & $162(40.3)$ & \multirow{2}{*}{$20.79^{* *}$} & $301(77.8)$ & $86(22.2)$ & \multirow{2}{*}{$4.63^{*}$} & $240(59.7)$ & $162(40.3)$ & \multirow{2}{*}{$20.79^{*}$} \\
\hline & & Problem & $278(75.1)$ & $92(24.9)$ & & $304(84.0)$ & $58(16.0)$ & & $278(75.1)$ & $92(24.9)$ & \\
\hline & \multirow{2}{*}{ Self-care } & Normal & $430(65.5)$ & $226(34.5)$ & \multirow{2}{*}{$4.64^{*}$} & $512(80.6)$ & $123(19.4)$ & \multirow{2}{*}{0.02} & $430(65.5)$ & $226(34.5)$ & \multirow{2}{*}{$4.64^{*}$} \\
\hline & & Problem & $90(75.6)$ & $29(24.4)$ & & $95(81.2)$ & $22(18.8)$ & & $90(75.6)$ & $29(24.4)$ & \\
\hline & \multirow{2}{*}{$\begin{array}{c}\text { Usual } \\
\text { Activities }\end{array}$} & Normal & $319(63.5)$ & $183(36.5)$ & \multirow{2}{*}{$8.14^{* *}$} & $385(79.5)$ & $99(20.5)$ & \multirow{2}{*}{1.2} & $319(63.5)$ & $183(36.5)$ & \multirow{2}{*}{$8.14^{*}$} \\
\hline & & Problem & 201(73.6) & $72(26.4)$ & & $222(82.8)$ & $46(17.2)$ & & 201(73.6) & $72(26.4)$ & \\
\hline & \multirow{2}{*}{$\begin{array}{c}\text { Pain/ } \\
\text { discomfort }\end{array}$} & Normal & $141(57.6)$ & $104(42.4)$ & \multirow{2}{*}{$14.79^{* *}$} & $194(81.5)$ & $44(18.5)$ & \multirow{2}{*}{0.14} & $141(57.6)$ & $104(42.4)$ & \multirow{2}{*}{$14.79^{*}$} \\
\hline & & Problem & $379(71.5)$ & $151(28.5)$ & & $413(80.3)$ & $101(19.6)$ & & $379(71.5)$ & $151(28.5)$ & \\
\hline & \multirow{2}{*}{$\begin{array}{l}\text { Anxiety/ } \\
\text { depression }\end{array}$} & Normal & $268(62.8)$ & $159(37.2)$ & \multirow{2}{*}{$7.94^{* *}$} & $331(80.5)$ & $80(19.5)$ & \multirow{2}{*}{0.41} & $268(62.8)$ & $159(37.2)$ & \multirow{2}{*}{$7.94^{*}$} \\
\hline & & Problem & $251(72.3)$ & $96(27.7)$ & & $276(81.2)$ & $64(18.8)$ & & $251(72.3)$ & $96(27.7)$ & \\
\hline
\end{tabular}

Independent effects of each variable on exercise were shown in Table 2. Exercisers were younger than non-exercisers in resistance exercise $(75.58+5.62$ vs. $76.90+5.88$ years, $p=0.014)$. Men were more likely to perform resistance exercise than women ( $47.2 \%$ vs. $26.0 \%, \mathrm{p}<.001)$. Body Mass Index does not independently affect any types of exercise participation. MMSE showed significant correlation with the three exercise types. Subjects who exercised moderate aerobic exercise $(\mathrm{t}=-2.925, \mathrm{p}=0.004)$, resistance exercise 2 times per week $(\mathrm{t}=3.221, \mathrm{p}<.001)$, and walking $(\mathrm{t}=-2.925$, $\mathrm{p}=0.004$ ) had significantly higher MMSE score than non-exercisers.

Each EQ-5D subset showed significant correlations with the three exercise types. Subjects with problems in the mobility dimensions performed less of all exercise types than those with normal mobility (Moderate aerobic: $24.9 \%$ vs. 75.1\%, p<.001; 
Resistance exercise: $16.0 \%$ vs. $84.0 \%$, $\mathrm{p}=0.020$; walking: $24.9 \%$ vs. $75.1 \%, \mathrm{p}<.001)$.

Subjects with problems in the self-care dimensions performed less of moderate aerobic exercise and walking than those with normal self-care (Moderate aerobic: $24.4 \%$ vs. $75.6 \%$, $\mathrm{p}=0.019$; Walking: $24.4 \%$ vs. $75.6 \%, p=0.019$ ). Subjects with problems in usual activity dimensions performed less of moderate aerobic exercise and walking than those with normal, usual activity (Moderate aerobic: $26.4 \%$ vs. $73.6 \%$, $\mathrm{p}=0.003$; Walking: $26.4 \%$ vs. $73.6 \%, p=0.003)$. Subjects with problems in anxiety/depression dimensions performed less of moderate aerobic exercise and walking than those with normal anxiety/depression (Moderate aerobic: $27.7 \%$ vs. $72.3 \%, p=0.003$; walking: $27.7 \%$ vs. $72.3 \%$, $\mathrm{p}=0.003$ ) (Table 2).

Table 3: Adjusted odds ratios for the three exercise types

\begin{tabular}{|c|c|c|c|c|}
\hline \multirow{2}{*}{ Variables } & \multirow{2}{*}{ Categories } & Aerobic EXS & \multirow{2}{*}{$\begin{array}{c}\text { Resistance EXS } \\
\text { Adjusted OR (95\%CI) }\end{array}$} & \multirow{2}{*}{$\begin{array}{c}\text { Walking } \\
\text { Adjusted OR (95\%CI) }\end{array}$} \\
\hline & & Adjusted OR (95\%CI) & & \\
\hline \multicolumn{5}{|l|}{ EQ-5D } \\
\hline \multirow{2}{*}{ Mobility } & Normal & 1 & 1 & 1 \\
\hline & Problem & $0.888(0.630-1.252)$ & $0.633(0.457-0.877)$ & $0.538(0.396-0.732)$ \\
\hline \multirow{2}{*}{ Self-care } & Normal & 1 & 1 & 1 \\
\hline & Problem & $0.726(0.433-1.217)$ & $0.987(0.950-1.500)$ & $0.551(0.367-0.827)$ \\
\hline \multirow{2}{*}{ Usual Activities } & Normal & 1 & 1 & 1 \\
\hline & Problem & $0.824(0.571-1.189)$ & $0.905(0.644-1.272)$ & $0.715(0.521-0.980)$ \\
\hline \multirow{2}{*}{ Pain/discomfort } & Normal & 1 & 1 & 1 \\
\hline & Problem & $0.701(0.490-1.004)$ & $0.920(0.654-1.295)$ & $0.768(0.552-1.070)$ \\
\hline \multirow{2}{*}{ Anxiety/depression } & Normal & 1 & 1 & 1 \\
\hline & Problem & $0.897(0.637-1.265)$ & $0.758(0.548-1.048)$ & $0.641(0.473-0.868)$ \\
\hline
\end{tabular}

The most important findings of this study were that not all types of exerciser showed fewer problems in all EQ-5D dimensions than non-exercisers in older people with MCI. Among the QOL dimension, the mobility dimension was significantly associated with resistance and walking exercises, whiles the self-care, usual activity; anxiety/depression was associated only with walking after controlling for covariates. Older people with MCI were more likely to perform walking exercise, followed by resistance exercise and moderate aerobic exercise that is ACSM recommended. This result confirmed previous research that walking could be considered a valuable method to improve exercise tolerance and quality of life in MCI and Alzheimer's El-Kader et al. [13].

Previous research reported that brisk walking would be considered a valuable method to improve quality of life in MCI and Alzheimer's Wetzels, Zuidema, et al. [14]. Other research also reported impaired cognitive, and mobility critically impact older adults' QOL Davis, Bryan, et al. [15]. However, a few studies reported the association between specific types of exercise and different domain of EQ-5D. In this study, after controlling for all covariates, a moderate intensity aerobic exercise more than 150 minutes per week was not independently associated with any

\section{Discussion}

Adjusted odds ratios for the three exercise types analyzed by multivariate logistic regression were shown in Table 3. After controlling for gender, age, MMSE, BMI, subjects with problems in mobility dimension performed less exercise of resistance and 95\% CI: 0.457-0.877; walking: OR, 0.538; 95\% CI: 0.396-0.732). Subjects with problems in self-care performed less walking than those with normal self-care (Or, 0.551; 95\% CI: 0.367-0.827). Subjects with problems in a usual activity performed less walking than those with normal self-care (OR, 0.715; 95\% CI: 0.521-0.980). Subjects with problems in anxiety/depression performed less walking than those with normal anxiety/depression (OR, 0.641; 95\% CI: 0.473-0.868) (Table 3). walking than those with normal mobility (resistance: 0R,0.633;

dimension of QOL. However, resistance and walking exercise were independently associated with mobility dimension, and walking was correlated with all EQ-5D dimensions. The results of the current study are similar results of Oh et al. [16] study in general older adult population in that walking exercise was independently associated with two QOL dimensions (mobility and self-care), while resistance exercise was correlated with only the QOL dimension of mobility.

Because of the QOL dimension of self-care, usual activities, pain/discomfort, anxiety/depression do not require much muscle strength, walking alone might have had effects on the QOL dimension. In the current study, moderate intensity aerobic exercise was not associated with any of EQ-5D dimension. This may be caused by the misunderstanding of the question of 'moderate' intensity of aerobic exercise for the elders with MCI. Another reason may be a different preference for the exercise of seniors with MCI from general seniors. The exercise participation for moderate intensity aerobic was lower (25.2\% for men, $24.75 \%$ for women) than that of general seniors (32.3\% for men and $25.9 \%$ for women) analyzing the fifth Korea National Health and Nutrition Examination Surveys Kim et al. [17]. For older adults who have progressed from normal to mild cognitive decline to a diagnosis of MCI, participation in the 
regular moderate intensity of aerobic exercise may be difficult. This may be caused to underestimate the prevalence of moderate aerobic participation but rather increase the prevalence of regular walking.

Further, individuals with MCI experience changes in the psychological function such as depression, anxiety, agitation Apostolova \& Cummings [18] as well as quality of life Teng et al. [19]. These changes in psychological and cognitive function may be more influential on the sub-dimension of EQ-5D. This study has several limitations. Study participants were recruited by social workers with a convenient sampling. As such, the results of this study may not be generalizable to other risk older populations. In conclusion, regular resistance exercise and walking exercise were associated QOL. Specifically, the mobility was independently associated with resistance exercise, and the mobility, self-care, usual activity, and anxiety/ depression were independently associated with walking exercise in this older with MCI. Given that both impaired cognitive function and reduced mobility contributed to the loss of functional independence which is associated with reduced quality of life, this result suggests that engaging in regular walking and resistance exercise is essential for achieving higher QOL in the elders with MCI. Further investigation is needed for the contribution of the frequency and intensity of specific exercise type for MCI population.

\section{References}

1. Hood A (2013) The Study of Global Aging: A Smart Move. Generations 37(1): 4 .

2. Petersen RC, R Doody, Kurz A, Mohs RC, Morris JC, et al. (2001) Current concepts in mild cognitive impairment. Archives of neurology 58(12): 1985-1992.

3. Jia J, A Zhou, Wei C, Jia X, Wang F, et al. (2014) The prevalence of mild cognitive impairment and its etiological subtypes in elderly Chinese. Alzheimer's \& Dementia 10(4): 439-447.

4. Lara E, A Koyanagi, Olaya B, Lobo A, Miret M, et al. (2016) Mild cognitive impairment in a Spanish representative sample: prevalence and associated factors. International journal of geriatric psychiatry 31(8): 858-867.

5. Limongi F, P Siviero, Noale M, Gesmundo A, Crepaldi G, et al. (2017) Prevalence and conversion to dementia of Mild Cognitive Impairment in an elderly Italian population. Aging Clinical and Experimental Research p. 1-10.

6. Smith PJ, JA Blumenthal, Hoffman BM, Cooper H, Strauman TA, et al. (2010) Aerobic exercise and neurocognitive performance: a meta- analytic review of randomized controlled trials. Psychosomatic medicine 72(3): 239.

7. Paillard-Borg S, L Fratiglioni, Xu W, Winblad B, Wang HX (2012) an active lifestyle postpones dementia onset by more than one year in very old adults. Journal of Alzheimer's disease 31(4): 835-842.

8. Nascimento C, J Pereira, De Andrade LP, Garuffi M, Talib LL, et al. (2014) Physical exercise in MCI elderly promotes reduction of pro-inflammatory cytokines and improvements on cognition and BDNF peripheral levels. Current Alzheimer Research 11(8): 799-805.

9. Chen CH, YJ Chen, Tu HP, Huang MH, Jhong JH, et al. (2014) Benefits of exercise training and the correlation between aerobic capacity and functional outcomes and quality of life in elderly patients with coronary artery disease. The Kaohsiung journal of medical sciences 30(10): 521530.

10. Lautenschlager NT, KL Cox, Flicker L, Foster JK, Van Bockxmeer FM, et al. (2008) Effect of physical activity on cognitive function in older adults at risk for Alzheimer disease. JAMA: the journal of the American Medical Association 300(9): 1027-1037.

11. Park JH, Kwon YC (1989) Standardization of Korean version of the Mini-Mental State Examination for use in the elderly. Part II: Diagnostic validity. J Korean Neuropsychiatric Assoc 28: 508-513.

12. EQ-5D (2016) an instrument to describe and value health.

13. El-Kader SMA (2011) Role of aerobic exercise training in changing exercise tolerance and quality of life in Alzheimer's. European Journal of General Medicine 8(1): 1-6.

14. Wetzels R, SU Zuidema, De Jonghe JF, Verhey FR, Koopmans RT (2010) Determinants of quality of life in nursing home residents with dementia. Dementia and geriatric cognitive disorders 29(3): 189-197.

15. Davis JC, S Bryan, Rob McLeod, Jessica Rogers, Karim Khan, et al. (2012) Exploration of the association between quality of life, assessed by the EQ-5D and ICECAP-0, and falls risk, cognitive function and daily function, in older adults with mobility impairments. BMC geriatrics 12(1): 65 .

16. Sang-Ho Oh, Don-Kyu Kim, Shi-Uk Lee, Se Hee Jung, Sang Yoon Lee (2017) Association between exercise type and quality of life in a community-dwelling older people: A cross-sectional study. PloS one 12(12): e0188335.

17. Kim J, Y Lee, Kye S, Chung YS, Kim KM (2015) Association between healthy diet and exercise and greater muscle mass in older adults. Journal of the American Geriatrics Society 63(5): 886-892.

18. Apostolova LG, JL Cummings (2008) Neuropsychiatric manifestations in mild cognitive impairment: a systematic review of the literature. Dementia and geriatric cognitive disorders 25(2): 115-126.

19. Teng E, K Tassniyom, Lu PH (2012) reduced quality-of-life ratings in mild cognitive impairment: analyses of subject and informant responses. The American Journal of Geriatric Psychiatry 20(12): 1016-1025.
This work is licensed under Creative Commons Attribution 4.0 License

Submission Link: http://biomedres.us/submit-manuscript.php

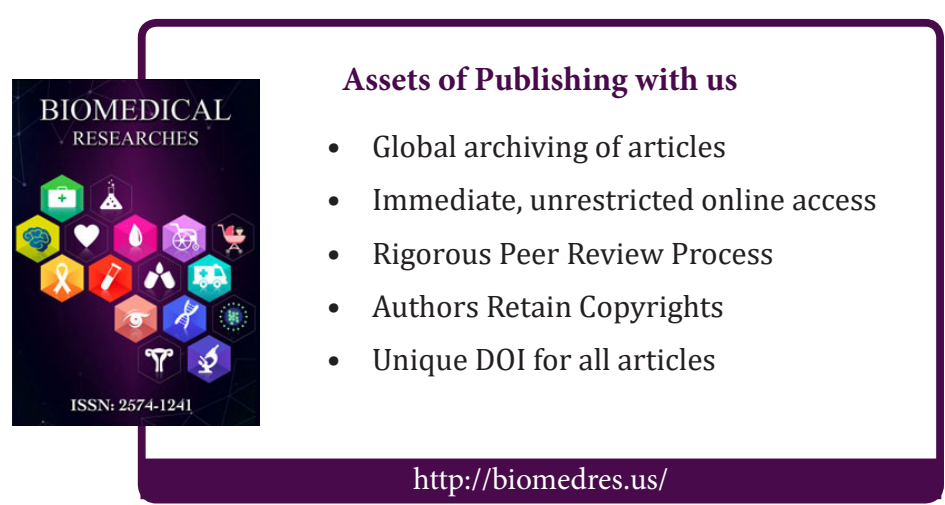

